\title{
The Effect of Psychological Hotwash on Resilience of Emergency Medical Services Personnel
}

\author{
Abbasali Ebrahimian $\mathbb{D D}^{1,2}$ Seyed-Mahdi Esmaeili ${ }^{1}{ }^{3},{ }^{3}$ Arash Seidabadi $\mathbb{D}^{4},{ }^{4}$ \\ and Ali Fakhr-Movahedi ${ }^{2}{ }^{2}$ \\ ${ }^{1}$ Clinical Research Development Center, Shahid Beheshti Hospital, Qom University of Medical Sciences, Qom, Iran \\ ${ }^{2}$ Nursing Care Research Center, Semnan University of Medical Sciences, Semnan, Iran \\ ${ }^{3}$ Student Research Committee, Nursing School, Semnan University of Medical Sciences, Semnan, Iran \\ ${ }^{4}$ Emergency Medicine Department, Shahroud University of Medical Sciences, Shahroud, Iran
}

Correspondence should be addressed to Ali Fakhr-Movahedi; alimovi49@semums.ac.ir

Received 2 June 2021; Revised 23 July 2021; Accepted 11 August 2021; Published 21 August 2021

Academic Editor: Yan-Ren Lin

Copyright ( $\odot 2021$ Abbasali Ebrahimian et al. This is an open access article distributed under the Creative Commons Attribution License, which permits unrestricted use, distribution, and reproduction in any medium, provided the original work is properly cited.

\begin{abstract}
Introduction. Emergency medical services (EMS) personnel are exposed to stress. Job stress in EMS personnel can reduce their resilience and have adverse effects on their clinical performance and mental health, thus reducing the quality of their work. The present research was performed to determine the effect of psychological hotwash on resilience of emergency medical services personnel. Methods. This study was a quasiexperimental. Sixty-four EMS personnel were randomly divided into two groups of hotwash and control. The psychological hotwash program was performed in the intervention group for a month based on the protocol; however, the control group continued their usual work and received no intervention. A day and six weeks after the psychological hotwash in the intervention group, the resilience of the EMS personnel was remeasured in both groups. Results. Before the intervention, the participants' mean resilience score was $138.37 \pm 7.04$ in the intervention group and $137.34 \pm 8.48$ in the control group. There was a statistically significant difference between the mean scores of resilience in the intervention and control groups a day after the intervention $(P=0.003)$. There was no statistically significant difference between the mean scores of resilience in the intervention and control groups 6 weeks after the intervention $(P=0.102)$. Conclusion. The EMS personnel's attendance at psychological hotwash sessions could increase their resilience. Nevertheless, the sessions should not be interrupted because the 6-week interruption of the sessions caused the nonsignificant scores of resilience in the hotwash and control groups. Hence, it is recommended to continue the investigation of the effects of hotwash on resilience, stress reduction, and job burnout reduction in EMS personnel by other researchers in different settings.
\end{abstract}

\section{Introduction}

Emergency medical services (EMS) is a society-based system that responds to the medical needs of casualties or patients with acute and emergency illnesses outside of healthcare centers and until transferring to a medical center $[1,2]$. EMS personnel are at high risk for mental disorders, stress, job burnout, depression, and posttraumatic stress disorder (PTSD) due to exposure to traumatic events and job stress [3-5]. In a systematic review, Petrie et al. (2018) indicated that the prevalence of PTSD, depression, and anxiety was
$11 \%, 15 \%$, and $15 \%$ in EMS personnel, respectively [6]. The prevalence of PTSD was reported as $50.21 \%$ in the Iranian EMS personnel [7], which is much higher than the world average.

Studies indicate that EMS personnel are exposed to stress due to the embodiment of their own death, complexity of patients' clinical conditions, interruption of EMS provision, health hazards, interpersonal problems, interprofessional interactions, legal conflicts, dependence on patients, and exposure to unsafe situations [8,9]. Job stress in EMS personnel can reduce their resilience and have adverse 
effects on their clinical performance and mental health, thus reducing the quality of their work $[10,11]$. Hence, it is necessary to reduce stress and increase resilience in EMS personnel.

Resilience has become more important in the last two decades. It enables people to control life stressors. It is also a successful adaptation to adverse environmental conditions [12]. Studies indicate that EMS personnel resilience is not desirable, and it can be affected by factors, such as secondary traumatic stress, resilience, posttraumatic growth, and changes in outlook, grit, and stress [13-15].

Analgesics, sedatives, acupuncture and acupressure, yoga, massage therapy, spirituality enhancement, and aromatherapy are usually used to reduce stress and increase resilience against some mental disorders [16-18]. However, some of these methods are associated with side effects, and others may be unwelcome by EMS personnel. The need assessments indicate that EMS personnel require easier access to mental health and improved interemployee relationships [4]. Studies also indicate that EMS personnel use talking with colleagues and inner dialogue strategies to deal with job burnout and increase resilience in stressful situations $[19,20]$. It implies that EMS personnel tend to discuss their problems with other people to resolve their internal conflicts. Therefore, it seems that the use of debriefing known as psychological hotwash in the field of accidents and disasters can reduce stress and increase resilience in EMS personnel.

Psychological debriefing is a method of preventing the onset of PTSD symptoms in various injuries using approaches, such as normalizing responses, modulating emotions, and the way of dealing with symptoms of the disorder in a group session [21]. The method was first used during World War II. It was then developed by Jeffery Mithell in 1983 to manage stress in critical events and termed Critical Incident Stress Debriefing (CISD) [22]. CISD has been used in several studies to reduce PTSD, increase the quality of life, and improve clinical performance $[23,24]$. However, the extent of its impact on the resilience of EMS personnel is not clear. Therefore, the present research was performed to determine the effect of psychological hotwash on resilience of emergency medical services personnel.

\section{Methods}

2.1. Study Design, Setting, and Participants. The research was a quasiexperimental study on EMS personnel of Shahroud in Semnan province, Iran, as the statistical population. The urban population was 218628 people during sampling, and their emergency measures were carried out by 20 EMS stations. There were 7 to 9 technicians working in each EMS station. To select the participants in the study, we first wrote the names of these 20 stations on separate papers and placed them in a small cloth bag. A 7-year-old child was then asked to take out 12 pieces of paper separately from the bag. The station's name on the first paper was placed in the intervention group and the station's name on the second paper was put in the control group. Using this method, 6 stations were considered in the intervention group and 6 in the control group. Moreover, EMS technicians working in the EMS stations with the inclusion criteria were included in the study as a research sample.

Inclusion criteria are as follows:

(1) Take care of at least one emergency patient for 24 hours before attending the hotwash session

(2) One-year experience in EMS

(3) Do not have known mental and physical disorders

Exclusion criteria are as follows:

(1) Unwillingness to continue participating in this research

(2) Absence from attending 8 sessions of psychological hotwashing

2.2. Sample Size Estimation. To estimate the sample size, the first two groups of 5 individuals were selected in a pilot study, for which the study protocol was implemented. Then, the mean \pm standard deviation of the difference in resilience scores in the pretest stage was calculated and compared with the scores of 4 weeks and 6 weeks later. The obtained values were inserted into G-Power 3.1.9 considering a 5\% alpha, $80 \%$ power, and an effect size of 0.77 . Using this method, the necessary sample size was 28 per group. As it was necessary to include all personnel of a station in the study, we included more EMS personnel in the study.

2.3. Measures. The research tools in this study included demographic information questionnaires and an EMSRS questionnaire. Demographic information questionnaire included age, BMI, marital status, number of children, work experience, number of shifts per month, and number of night shifts per month. The EMSRS questionnaire had 31 questions to assess resilience in EMS personnel in Iran. The questionnaire assessed six factors of job motivation, communication challenges, social support, calmness at the incident scene, self-management or self-care, and consequences of stress. The questionnaire was designed as a 5 -point Likert-type scale ( $1=$ never, $2=$ rarely, 3 = sometimes, $4=$ often, and $5=$ always) and with a score range of 31 to 155 . The higher scores indicated more resilience, and lower scores revealed less resilience. The validity and reliability of the questionnaire were confirmed in the study of Ebadi et al. (Cronbach's alpha of 0.91 and intrapolar correlation coefficient of 0.85 ) [25].

2.4. Data Collection. Data collection was performed in the workplace of EMS personnel. To this end, qualified personnel in both groups individually responded to demographic information and EMSRS questionnaires without consulting with others. Thereafter, the psychological hotwash program was performed in the intervention group for a month based on the protocol; however, the control group continued their usual work and received no intervention. The psychological hotwash was done in the room of the director of Shahrud Medical Emergency and Accident Center at 8 AM every day 
and after the end of the night shift with the help of a psychologist. There were 7 to 10 participants in each session. Each of the eligible EMS personnel participated in at least 8 sessions of psychological hotwash. The first session lasted about 2 hours and between 70 and 90 minutes, later. A day and six weeks after the end of the one-month program of psychological hotwash in the intervention group, we remeasured the resilience of the EMS personnel in both groups. The 6-week reexamination was considered for the participants' resilience based on the results of Salyers et al. [26].

\subsubsection{Study Protocol. The study was performed within 7} stages:

(1) Preliminary stage: (a) acknowledgment of the presence of EMS personnel in session, (b) stating the objectives of the session, including training, quality improvement, and emotional processing, (c) explaining that the session was not a blame session, (d) explaining that the session lasted more than 60 minutes, and anyone could leave the session sooner for a personal reason, (e) creating a platform for all group members to speak and participate by asking them to introduce themselves; and (f) explaining that each participant can make suggestions to improve the quality of hotwash sessions.

(2) Reality stage: at this stage, the participants were asked to talk about what happened in their previous shift. The EMS manager and a psychologist helped participants to recall the events by asking additional questions.

(3) Thinking stage: at this stage, the participants talked about how to think about the problems that occurred in the previous shift and the meaning created by the problems in their minds.

(4) Reaction stage: the participants' emotional responses to the events that occurred in the previous shift were examined.

(5) Symptom stage: here, other reactions of the participants were examined (e.g. aggression, anger, change in appetite, headache, lethargy, confusion, lower accuracy, change in driving style, and sleep disturbance) to the events that occurred in the previous shift and their impact on their personal lives.

(6) Training stage: at this phase, the EMS center manager and psychologist taught the participants regarding the coping strategies and the way of proper response to incidents.

(7) Re-entry stage: at this stage, the process was reviewed quickly by the EMS manager or psychologist within 1 to 3 minutes, and the final result of the session was stated. The participants were asked to return to their normal lives [22].

2.5. Data Analysis. We entered data into the IBM SPSS version 16.0 software after collection and used descriptive statistics to indicate the frequency distribution, mean, and standard deviation of the data.
We used the independent $t$-test to compare age, BMI, number of children, work experience, number of shifts per month, and number of night shifts per month in the intervention and control groups. The chi-square test was used to compare marital status in the experimental and control groups.

The independent $t$-test was used to compare the resilience status between the intervention and control groups before and after the intervention and 6 weeks after the intervention. The significance level of all tests was 0.05 .

2.6. Ethical Consideration. This study was approved by the Ethical Committee of Semnan University of Medical Sciences (Cod: IR.SEMUMS.REC.1398.240). Necessary coordination was made with the incident manager and the officials of the sampling sites. Then, the working method was explained to all EMS employees, and informed consent was obtained from them to participate in the research.

\section{Results}

Among 89 EMS technicians, 71 met the inclusion criteria. However, 7 were excluded from the study for different reasons, such as not participating in 8 hotwash sessions $(n=5)$ and unwillingness to continue participating in the study $(n=2)$. Totally, 64 participants were randomly divided into two groups of intervention $(n=32)$ and control $(n=32)$, and their data were analyzed (Figure 1).

All participants in the study were male because all emergency medical technicians are male in Iran. The mean age of the participants was $35.00 \pm 6.15$. The minimum and maximum ages of the participants were 22 and 35 years, respectively. The participants' $\mathrm{BMI}$ was within the range of 18.90 to 33.95 and with an average of $25.36 \pm 3.21$. Among the participants, $82.80 \%$ were married and the rest were single. The EMS personnel's work experience was between 2 and 25 years with an average of $10.55 \pm 5.29$. The participants' average number of shifts per month was $17.58 \pm 3.00$, and the average number of night shifts was $8.61 \pm 1.76$. The average number of emergency missions was $10.68 \pm 2.96$ during the study. There was a statistically significant difference between age and BMI of the participants in the two groups $(P>0.05)$; however, the two groups were homogeneous in terms of marital status, work experience, number of shifts per month, number of night shifts per month, and number of emergency missions during the study $(P>0.05)$ (Table 1).

Before the intervention, the participants' mean resilience score was $138.37 \pm 7.04$ in the intervention group and $137.34 \pm 8.48$ in the control group. The statistical independent $t$-test indicated that there was no statistically significant difference between mean scores of resilience in the intervention and control groups before the intervention $(P=0.498)$. A day after the end of the interventions, the participants' mean score of resilience was $140.71 \pm 8.39$ and $135.15 \pm 6.05$ in the intervention and the control groups, respectively. There was a statistically significant difference between the mean scores of resilience in the intervention and 


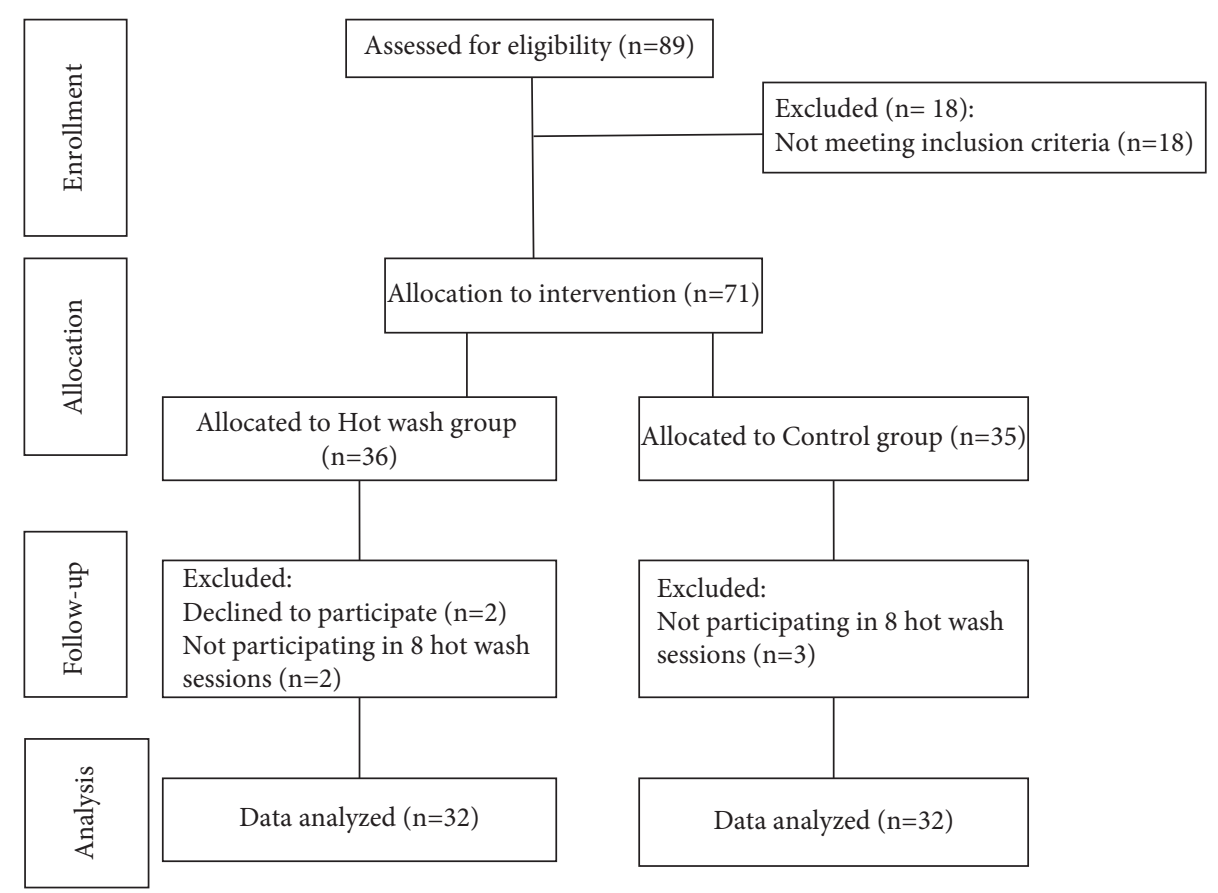

FIgURE 1: The flow of patients through the trial.

TABle 1: Demographic and working status of psychological hotwash and control groups.

\begin{tabular}{|c|c|c|c|c|}
\hline \multirow{2}{*}{ Variables } & \multicolumn{3}{|c|}{ Frequency in groups } & \multirow{2}{*}{$P$ value } \\
\hline & Hotwash & Control & Total & \\
\hline Age (years), mean $\pm \mathrm{SD}$ & $36.63 \pm 6.03$ & $33.38 \pm 5.92$ & $35.00 \pm 6.15$ & $0.034^{\mathrm{b}}$ \\
\hline $\mathrm{BMI}$, mean $\pm \mathrm{SD}$ & $26.41 \pm 2.99$ & $24.33 \pm 3.12$ & $25.36 \pm 3.21$ & $0.003^{\mathrm{b}}$ \\
\hline \multicolumn{5}{|l|}{ Marriage status, $n(\%)$} \\
\hline Married & $29(90.62)$ & $24(75)$ & $53(82.8)$ & \multirow{2}{*}{$0.098^{\mathrm{C}}$} \\
\hline Single & $3(9.38)$ & $8(25)$ & $11(17.2)$ & \\
\hline Work experience (years), mean $\pm S D$ & $12.06 \pm 5.81$ & $9.03 \pm 4.29$ & $10.5 \pm 5.29$ & $0.086^{\mathrm{b}}$ \\
\hline Number of shifts per month, mean $\pm S D$ & $17.28 \pm 3.00$ & $17.88 \pm 3.02$ & $17.58 \pm 3.00$ & $0.385^{\mathrm{b}}$ \\
\hline Number of night shifts per month, mean \pm SD & $8.22 \pm 1.21$ & $9.00 \pm 2.12$ & $8.61 \pm 1.76$ & $0.097^{\mathrm{b}}$ \\
\hline Number of missions during the study, mean $\pm \mathrm{SD}$ & $80.56 \pm 10.31$ & $79.06 \pm 10.13$ & $80.20 \pm 10.18$ & $0.110^{\mathrm{b}}$ \\
\hline
\end{tabular}

Data are presented as $n(\%)$, or mean $\pm \mathrm{SD}$. SD: standard deviation. ${ }^{\mathrm{b}}$ Independent samples $t$-test. ${ }^{\mathrm{c}} \mathrm{Chi}$-square test.

control groups a day after the intervention $(P=0.003)$. The difference in resilience score changes was $5.22 \pm 1.81$ in the hotwash group and $2.18 \pm 4.63$ in the control group before and a day after the intervention. There was a statistically significant difference between the differences in the mean resilience scores of the intervention and control groups a day after the intervention $(P=0.002)$ (Table 2).

Six weeks after the end of the interventions, the participants' mean score of resilience was $142.68 \pm 7.58$ and $139.68 \pm 6.87$ in the intervention and the control groups, respectively. There was no statistically significant difference between the mean scores of resilience in the intervention and control groups 6 weeks after the intervention $(P=0.102)$. The difference in resilience score changes was $3.787 \pm 4.13$ in the hotwash group and $2.34 \pm 4.17$ in the control group before and six weeks after the intervention. There was no statistically significant difference between the differences in the mean resilience scores of the intervention and control groups six weeks after the intervention $(P=0.171)$ (Table 2).
There was a significant difference between mean scores of job motivation, self-management, and social support subscales before and a day after the intervention $(P \leq 0.05)$. There was also a significant difference between the mean scores of the social support subscale before and six weeks after the intervention $(P \leq 0.05)$ (Table 3$)$.

\section{Discussion}

In recent years, increasing resilience has attracted the focus of disaster managers [27]. EMS personnel experience a huge deal of stress while caring for patients, which can affect their clinical performance and decisions [14]. Increasing resilience can reduce stress, delay job burnout, and increase EMS personnel efficiency $[13,28]$. Psychological hotwash is a way to help EMS personnel increase their resilience to job stress. The aim of this study was to determine the effect of psychological hotwash on resilience of emergency medical services personnel. 
TABLE 2: Comparison of the resiliency score before and after interventions in the psychological hotwash and control groups.

\begin{tabular}{|c|c|c|c|c|c|c|}
\hline \multirow{3}{*}{ Times } & \multirow{3}{*}{ Groups } & \multicolumn{2}{|c|}{ Resiliency } & \multirow{3}{*}{$t$} & \multirow{3}{*}{$d f$} & \multirow{3}{*}{$P$ value } \\
\hline & & & & & & \\
\hline & & Mean & $\mathrm{SD}$ & & & \\
\hline \multirow{2}{*}{ Before intervention } & Hotwash & 138.90 & 9.81 & \multirow{2}{*}{0.681} & \multirow{2}{*}{62} & \multirow{2}{*}{$0.498^{\mathrm{a}}$} \\
\hline & Control & 137.34 & 8.47 & & & \\
\hline \multirow[b]{2}{*}{ One day after the intervention } & Hotwash & 140.71 & 8.39 & \multirow{2}{*}{3.040} & \multirow{2}{*}{62} & \multirow{2}{*}{$0.003^{\mathrm{a}}$} \\
\hline & Control & 135.15 & 6.05 & & & \\
\hline \multirow{2}{*}{ Six weeks after the intervention } & Hotwash & 142.68 & 7.55 & \multirow{2}{*}{1.660} & \multirow{2}{*}{62} & \multirow{2}{*}{$0.102^{\mathrm{a}}$} \\
\hline & Control & 139.68 & 6.87 & & & \\
\hline \multirow{2}{*}{ The difference between resilience before the intervention and one day after the intervention } & Hotwash & 1.81 & 5.22 & \multirow{2}{*}{-3.23} & \multirow{2}{*}{62} & \multirow{2}{*}{$0.002^{\mathrm{a}}$} \\
\hline & Control & -2.18 & 4.63 & & & \\
\hline \multirow{2}{*}{ The difference between resilience before the intervention and six weeks after the intervention } & Hotwash & 3.78 & 4.13 & \multirow{2}{*}{-1.38} & \multirow{2}{*}{62} & \multirow{2}{*}{$0.171^{\mathrm{a}}$} \\
\hline & Control & 2.34 & 4.17 & & & \\
\hline
\end{tabular}

${ }^{\mathrm{a}}$ Independent samples $t$-test.

TABLE 3: Comparison of the resiliency subscales mean scores before and after interventions in the psychological hotwash and control groups.

\begin{tabular}{|c|c|c|c|c|c|c|c|}
\hline \multirow{2}{*}{ Resiliency subscales } & \multirow{2}{*}{ Times } & \multirow{2}{*}{ Groups } & \multicolumn{2}{|c|}{ Resiliency score } & \multirow{2}{*}{$t$} & \multirow{2}{*}{$d f$} & \multirow{2}{*}{$P$ value } \\
\hline & & & Mean & $\mathrm{SD}$ & & & \\
\hline \multirow{6}{*}{ Job motivation } & \multirow{2}{*}{ Before intervention } & Hotwash & 58.62 & 5.02 & \multirow{2}{*}{0.354} & \multirow{2}{*}{62} & \multirow{2}{*}{0.725} \\
\hline & & Control & 58.21 & 4.11 & & & \\
\hline & \multirow{2}{*}{ One day after the intervention } & Hotwash & 60.50 & 2.99 & \multirow{2}{*}{2.103} & \multirow{2}{*}{62} & \multirow{2}{*}{0.040} \\
\hline & & Control & 58.68 & 3.84 & & & \\
\hline & \multirow{2}{*}{ Six weeks after the intervention } & Hotwash & 59.96 & 3.55 & \multirow{2}{*}{1.316} & \multirow[b]{2}{*}{62} & \multirow{2}{*}{0.193} \\
\hline & & Control & 58.75 & 3.85 & & & \\
\hline \multirow{6}{*}{ Communication challenges } & \multirow{2}{*}{ Before intervention } & Hotwash & 13.00 & 1.88 & \multirow{2}{*}{0.143} & \multirow{2}{*}{62} & \multirow{2}{*}{0.887} \\
\hline & & Control & 12.93 & 1.60 & & & \\
\hline & \multirow{2}{*}{ One day after the intervention } & Hotwash & 13.06 & 1.50 & & & \\
\hline & & Control & 13.34 & 1.40 & -0.774 & 62 & 0.442 \\
\hline & & Hotwash & 12.93 & 1.64 & & & \\
\hline & Six weeks after the intervention & Control & 13.37 & 1.43 & -1.135 & 62 & 0.261 \\
\hline & Before interyention & Hotwash & 9.00 & 1.16 & 257 & 62 & 0012 \\
\hline & Before intervention & Control & 8.25 & 1.16 & 2.57 & 62 & 0.012 \\
\hline & & Hotwash & 9.06 & 1.34 & & & \\
\hline Social support & One day after the intervention & Control & 8.40 & 0.97 & 2.234 & 62 & 0.029 \\
\hline & Six weeks after the intervention & Hotwash & 9.18 & 1.02 & 3110 & & \\
\hline & six weeks after the intervention & Control & 8.40 & 0.97 & 3.110 & 62 & 0.003 \\
\hline & & Hotwash & 22.56 & 1.96 & & & \\
\hline & Before intervention & Control & 22.43 & 2.09 & 0.246 & 62 & 0.806 \\
\hline & & Hotwash & 23.53 & 1.52 & & & \\
\hline Remainıng calm & One day after the intervention & Control & 23.31 & 1.20 & 0.637 & 62 & 0.526 \\
\hline & & Hotwash & 23.71 & 1.46 & & & \\
\hline & Six weeks after the intervention & Control & 23.21 & 1.28 & 1.450 & 62 & 0.152 \\
\hline & & Hotwash & 22.43 & 1.99 & & & \\
\hline & Before intervention & Control & 21.96 & 1.63 & 1.027 & 62 & 0.309 \\
\hline & & Hotwash & 22.93 & 1.50 & & & \\
\hline Selt-management & One day atter the intervention & Control & 22.31 & 1.51 & 1.659 & 62 & 0.102 \\
\hline & Six weelse fter the interyention & Hotwash & 22.96 & 1.46 & 1920 & 62 & 0050 \\
\hline & six weeks after the intervention & Control & 22.25 & 1.52 & 1.920 & 62 & 0.059 \\
\hline & & Hotwash & 13.28 & 1.57 & & & \\
\hline & Before intervention & Control & 13.53 & 1.68 & -0.614 & 62 & 0.541 \\
\hline Consequences of stress & One day after the intervention & Hotwash & 13.96 & 1.03 & & & \\
\hline Consequences or stress & One day after the intervention & Control & 13.68 & 1.67 & 0.809 & 62 & 0.422 \\
\hline & Six weeks af & Hotwash & 13.90 & 1.17 & 0.605 & 62 & 0.547 \\
\hline & six weeks arter the intervention & Control & 13.68 & 1.67 & 0.605 & 02 & $0.54 /$ \\
\hline
\end{tabular}


In this study, there was a statistically significant difference between the mean scores of resilience in the intervention and control groups a day after the end of the intervention. However, there was no significant difference between the mean scores of resilience in the intervention and control groups 6 weeks after interventions. The finding indicated that the degree of resilience increased in the EMS personnel attending hotwash sessions at least 8 times per month. However, if attending hotwash sessions was stopped for 6 weeks, the effect of hotwash on resilience would stop. Froutan et al. in a study found that a stress management program could reduce anxiety and increase EMS personnel's resilience [29]. Arce Edgar Carlos also found that the use of debriefing was effective in reducing the early respondents' stress [30]. Bohström et al. indicated that discussions with colleagues directly could reduce stress in EMS personnel [31]. In contrast, Woods and Ginger Lee argued that the EMS personnel's attendance at hotwash sessions did not help to treat PTSD symptoms and could even make it worse. He mentioned that the EMS personnel's attendance only in a debriefing session and using only a debriefing technique can worsen the symptoms of PTSD [32]. Devilly and Cotton also indicated that the use of debriefing was not appropriate to reduce workplace stress [33]. It seems that the noneffect of debriefing on stress in other studies was probably caused by the implementation of the debriefing protocol and its duration. Only a debriefing session was held in studies where debriefing did not affect stress. However, in this study, the debriefing sessions lasted for a month, and the EMS personnel attended at least 8 debriefing sessions per month. In addition to the presence of a psychologist, the manager of the Accident and Emergency Medical Center of the province also attended the session in our study. Therefore, it is suggested to continue the sessions and the presence of the EMS senior manager in the sessions for higher effectiveness of the debriefing protocol to reduce stress and increase resilience in EMS personnel. It is also recommended that a debriefing session be held after each heavy mission to reduce the stress of prehospital emergency personnel.

\section{Conclusion}

The EMS personnel's attendance at psychological hotwash sessions could increase their resilience. Nevertheless, the sessions should not be interrupted because the 6-week interruption of the sessions caused the nonsignificant scores of resilience in the hotwash and control groups. Hence, is recommended to continue the investigation of the effects of hotwash on resilience, stress reduction, and job burnout reduction in EMS personnel by other researchers in different settings.

\section{Data Availability}

The data used to support the findings of this study are available from the corresponding author upon request.

\section{Conflicts of Interest}

The authors declare that there are no conflicts of interest.

\section{Acknowledgments}

The authors would like to thank the Nursing Care Research Center of Semnan University of Medical Sciences for providing facilities for this work and all EMS personals that participated in this study and their sincere cooperation. This study was a part of a study supported financially by Semnan University of Medical Sciences (grant no. 1646).

\section{References}

[1] C. P. Z. Foo, M. Ahghari, and R. D. MacDonald, "Use of geographic information systems to determine new helipad locations and improve timely response while mitigating risk of helicopter emergency medical services operations," Prehospital Emergency Care, vol. 14, no. 4, pp. 461-468, 2010.

[2] C. Elmqvist, B. Fridlund, and M. Ekebergh, "More than medical treatment: the patient's first encounter with prehospital emergency care," International Emergency Nursing, vol. 16, no. 3, pp. 185-192, 2008.

[3] I. C. Ma, W. H. Chang, C.-L. Wu, and C.-H. Lin, "Risks of post-traumatic stress disorder among emergency medical technicians who responded to the 2016 Taiwan earthquake," Journal of the Formosan Medical Association, vol. 119, no. 9, pp. 1360-1371, 2020.

[4] M. P. Kling, Needs Assessment for Mental Health Support towards Emergency Medical Service (EMS) Personnel, Regent University, Virginia Beach, VA, USA, 2021.

[5] M. Y. Choi, "Mental and physical factors influencing wellbeing among south Korean emergency workers," International Journal of Environmental Research and Public Health, vol. 18, no. 1, p. 70, 2021.

[6] K. Petrie, J. Milligan-Saville, A. Gayed et al., "Prevalence of PTSD and common mental disorders amongst ambulance personnel: a systematic review and meta-analysis," Social Psychiatry and Psychiatric Epidemiology, vol. 53, no. 9, pp. 897-909, 2018.

[7] M. Golitaleb, A. Mozafari, K. Yousefi, M. Harorani, and A. Sahebi, "Post-traumatic stress disorder in emergency medical services personnel in Iran: a letter to the editor," Iranian Journal of Psychiatry and Behavioral Sciences, vol. 14, no. 1, Article ID e100281, 2020.

[8] A. P. Barbee, M. E. Fallat, R. Forest, M. E. McClure, K. Henry, and M. R. Cunningham, "EMS perspectives on coping with child death in an out-of-hospital setting," Journal of Loss \& Trauma, vol. 21, no. 6, pp. 455-470, 2016.

[9] A. Afshari, S. R. Borzou, F. Shamsaei, E. Mohammadi, and L. Tapak, "Perceived occupational stressors among emergency medical service providers: a qualitative study," BMC Emergency Medicine, vol. 21, no. 1, p. 35, 2021.

[10] Z. Moshtagh Eshgh, A. A. Aghaeinejad, A. Peyman, A. Amirkhani, and M. Chehregosha, "Relationship between occupational stress and mental health in male personnel of medical emergency in golestan province," Journal of Research Development in Nursing and Midwifery, vol. 12, no. 1, pp. 29-38, 2015.

[11] M. Bijani, S. Abedi, S. Karimi, and B. Tehranineshat, "Major challenges and barriers in clinical decision-making as perceived by emergency medical services personnel: a qualitative content analysis," BMC Emergency Medicine, vol. 21, no. 1, p. 11, 2021.

[12] S. R. Javadian, L. Khaleghi, and M. Fathi, "The effectiveness of emotional regulation training on negative affect and resilience of mothers with disabled children," Iranian Journal of 
Psychiatric Nursing (IJPN), vol. 6, no. 5, pp. 25-33, 2018, \{persion\}.

[13] C. L. Austin, M. Pathak, and S. Thompson, "Secondary traumatic stress and resilience among EMS," Journal of Paramedic Practice, vol. 10, no. 6, pp. 240-247, 2018.

[14] M. Musso, D. Tatum, D. Hamer, R. Hammarlund, L. Son, and P. McMahon, "The relationship between grit and resilience in emergency medical service personnel," The Ochsner Journal, vol. 19, no. 3, pp. 199-203, 2019.

[15] N. Ogińska-Bulik and M. Zadworna-Cieślak, "The role of resiliency and coping strategies in occurrence of positive changes in medical rescue workers," International Emergency Nursing, vol. 39, pp. 40-45, 2018.

[16] A. A. Ghods, N. Sotodehasl, M. Emadi khalaf, and M. Mirmohamadkhani, "Effects of lavender essential oil inhalation on nurses' job stress," Koomesh journal, vol. 19, no. 2 , pp. 421-428, 2017.

[17] M. R. Asgari, A. Vafaei-Moghadam, H. Babamohamadi, R. Ghorbani, and R. Esmaeili, "Comparing acupressure with aromatherapy using Citrus aurantium in terms of their effectiveness in sleep quality in patients undergoing percutaneous coronary interventions: a randomized clinical trial," Complementary Therapies in Clinical Practice, vol. 38, Article ID 101066, 2020.

[18] H. Babamohamadi, N. Sotodehasl, H. G. Koenig, F. Al Zaben, C. Jahani, and R. Ghorbani, "The effect of holy qur'an recitation on depressive symptoms in hemodialysis patients: a randomized clinical trial," Journal of Religion and Health, vol. 56, no. 1, pp. 345-354, 2017.

[19] M. N. Almutairi and A. A. El.Mahalli, "Burnout and coping methods among emergency medical services professionals," Journal of Multidisciplinary Healthcare, vol. 13, pp. 271-279, 2020.

[20] N. Avraham, H. Goldblatt, and E. Yafe, "Paramedics' experiences and coping strategies when encountering critical incidents," Qualitative Health Research, vol. 24, no. 2, pp. 194-208, 2014.

[21] G. Mirzazadeh, K. Nourozi, M. Fallahi, M. Vaziri, and T. Kiany, "Evaluating the effect of stressful incident debriefing on moral distress of nurses Iranian," Journal of Rehabilitation Research in Nursing (IJRN), vol. 2, no. 1, pp. 1-11, 2015, \{Persian\}.

[22] A. M. Mitchell, T. J. Sakraida, and K. Kameg, "Critical incident stress debriefing: implications for best practice," $D i$ saster Management \& Response, vol. 1, no. 2, pp. 46-51, 2003.

[23] M. R. Tuckey and J. E. Scott, "Group critical incident stress debriefing with emergency services personnel: a randomized controlled trial," Anxiety, Stress \& Coping, vol. 27, no. 1, pp. 38-54, 2014.

[24] D. O. Kessler, A. Cheng, and P. C. Mullan, "Debriefing in the emergency department after clinical events: a practical guide," Annals of Emergency Medicine, vol. 65, no. 6, pp. 690-698, 2015.

[25] A. Ebadi, R. Froutan, and J. Malekzadeh, "The design and psychometric evaluation of the emergency medical services resilience scale (EMSRS)," International emergency nursing, vol. 42, pp. 12-18, 2019.

[26] M. P. Salyers, C. Hudson, G. Morse et al., "BREATHE: a pilot study of a one-day retreat to reduce burnout among mental health professionals," Psychiatric Services, vol. 62, no. 2, pp. 214-217, 2011.

[27] C. Son, F. Sasangohar, T. Neville, S. C. Peres, and J. Moon, "Investigating resilience in emergency management: an integrative review of literature," Applied Ergonomics, vol. 87, Article ID 103114, 2020.

[28] H. Bruschwein and L. S. Gettle, "Multipronged intervention for reducing burnout and increasing resiliency in an interdisciplinary care team," BMJ Open Quality, vol. 9, no. 4, Article ID e001015, 2020.

[29] R. Froutan, N. Mahroughi, J. Malekzadeh, and S. R. Mazlom, "The effect of stress management training on anxiety and resilience of emergency medical staff," Ijpn, vol. 6, no. 3, pp. 46-53, 2018.

[30] E. C. Arce, The Efficacy of Debriefing for First Responders, California Southern University, Irvine, CA, USA, 2018.

[31] D. Bohström, E. Carlström, and N. Sjöström, "Managing stress in prehospital care: strategies used by ambulance nurses," International Emergency Nursing, vol. 32, pp. 28-33, 2017.

[32] Woods, G. L., "Post Traumatic Stress Symptoms and Critical Incident Stress Debriefing (CISD) in Emergency Medical Services (EMS) Personnel." (2007). Electronic Theses and Dissertations. Paper 2035.

[33] G. J. Devilly and P. Cotton, "Psychological debriefing and the workplace: defining a concept, controversies and guidelines for intervention," Australian Psychologist, vol. 38, no. 2, pp. 144-150, 2003. 